\title{
Light-based educational outreach activities for pre-university students
}

Shereen Hamdy, Takako Hirokawa, Philip Chan, Warren Jin, Victoria Rosborough, et al.

Shereen W. Hamdy, Takako Hirokawa, Philip Chan, Warren Jin, Victoria Rosborough, Eric Stanton, Andrew M. Netherton, Marilyn Garza, Wendy Ibsen, Demis D. John, John E. Bowers, "Light-based educational outreach activities for pre-university students," Proc. SPIE 11143, Fifteenth Conference on Education and Training in Optics and Photonics: ETOP 2019, 111432R (2 July 2019); doi: 10.1117/12.2523654

Event: Fifteenth Conference on Education and Training in Optics and Photonics: ETOP 2019, 2019, Quebec City, Quebec, Canada 


\title{
Light-based educational outreach activities for pre-university students
}

\author{
Shereen W. Hamdy ${ }^{\mathrm{a}}$, Takako Hirokawa ${ }^{\mathrm{a}}$, Philip Chan ${ }^{\mathrm{a}}$, Warren Jin ${ }^{\mathrm{a}}$, Victoria Rosborough ${ }^{\mathrm{a}}$, \\ Eric Stanton ${ }^{\mathrm{c}}$, Andrew M. Netherton ${ }^{\mathrm{a}}$, Marilyn Garza ${ }^{\mathrm{d}}$, Wendy Ibsen ${ }^{\mathrm{b}}$, Demis D. John ${ }^{\mathrm{a}}$, and \\ John E. Bowers ${ }^{\mathrm{a}}$ \\ ${ }^{a}$ Electrical and Computer Engineering Department, University of California, Santa Barbara, \\ CA, USA \\ ${ }^{\mathrm{b}}$ Center for Science and Engineering Partnerships, University of California, Santa Barbara, \\ CA, USA \\ ${ }^{\mathrm{c}}$ National Institute of Standards and Technology, Boulder, CO, USA \\ dSanta Barbara Junior High School, Santa Barbara, CA, USA
}

\begin{abstract}
We have developed three light-based educational outreach activities targeted towards pre-university students, emphasizing experiential hands-on components for core learning via challenges the students must complete. These activities leverage photonics concepts from two active research areas at the Univ. of California Santa Barbara: integrated optics and solid-state lighting. The activities center on (1) building a free-space optical link, (2) subtractive and additive color mixing, and (3) guiding light using gelatin "waveguides". These activities are self-contained that is, the necessary background and intuition are introduced and built, respectively, before culminating in the main demonstration. The color-mixing and gelatin waveguide activities were designed for middle school students (ages 10-13) and their families, while the free-space optical link activity was designed for high school students (ages 14-18). Graduate students, not necessarily in photonics or optics, typically lead these activities for groups of 20-30 students after an initial training. We have found that we are able to garner considerable excitement from students when activities culminate in a challenge, especially if it has a competitive nature. This allows leaders to emphasize important practices in scientific research, such as: using the success of others' experiments to one's benefit, making informed hypotheses and testing them, persistence in understanding and solving a problem, and finally, the desire to improve upon a working solution.
\end{abstract}

Keywords: education, outreach, photonics, optics, pre-university, high school, middle school, problem-based learning

\section{INTRODUCTION}

Santa Barbara is a well-known hub of photonics research, with several startup companies and larger companies conducting photonics research and development in the area, as well as many optics and photonics academic research groups at UC Santa Barbara. The Photonics Society at UC Santa Barbara, a student-led chapter of the IEEE Photonics Society, OSA, and SPIE, was formed in 2012 to connect students, academics, and professionals in the Santa Barbara area. One of the primary missions of the Photonics Society at UCSB is to conduct educational outreach serving communities in the Santa Barbara area, most often for middle and high school-aged students. As part of our outreach work, we often develop photonics-based educational activities. ${ }^{1}$

The Photonics Society at UCSB collaborates on educational outreach activities with university, student, and community organizations in the area, primarily with the Center for Science and Engineering Partnerships (CSEP), a center at UCSB that organizes educational programs to broaden participation and diversity in STEM fields. We have also worked with the Santa Barbara chapter of Girls Inc., a nonprofit organization that seeks to

Further author information: (Send correspondence to S.W.H.)

S.W.H.: E-mail: shereenwhamdy@ucsb.edu, Telephone: 18589457410

Fifteenth Conference on Education and Training in Optics and Photonics: ETOP 2019, edited by

Anne-Sophie Poulin-Girard, Joseph A. Shaw, Proc. of SPIE Vol. 11143, 111432R · (C) 2019

SPIE, ICO, IEEE, OSA · CCC code: 0277-786X/19/\$18 · doi: 10.1117/12.2523654 
equip girls with independence and skills to navigate economic, social, and gender barriers in the world, and the UCSB chapter of Mathematics, Engineering, and Science Achievement (MESA), an organization that supports underrepresented students in STEM education and careers through the educational pipeline.

Concepts relating to the fields of photonics and optics are commonly taught as part of a middle or high school curriculum, but often at a level that leaves room for additional concepts to be introduced at educational outreach events. For example, the California Next Generation Science Standards contain only one unit on light and waves for grades 6-8 and another for grades $9-12 .^{2}$ However, these concepts are closely related to everyday experiences that students are exposed to from a young age, such as light, color, waves, reflection, communication, and the internet. This creates opportunities for the development of educational outreach activities and demonstrations that take advantage of students' experiences and pre-existing knowledge to teach new concepts not often taught in school, and to inspire curiosity in optics and photonics study and careers.

In this paper, we describe three educational outreach activities we have developed based on concepts in optics and photonics, and we explain our experiences and findings from our development and deployment of these activities.

\section{ACTIVITY DEVELOPMENT}

We aimed to design outreach activities that would educate pre-university-aged students on concepts from optics and photonics while connecting these concepts to the real world and students' personal experiences. Our hope is that these activities stimulate students' curiosity in science and STEM careers, particularly in photonics. We also designed the activities to be accessible to students and self-contained. Lastly, we wanted to strike a good balance between the amount of material we included and keeping the pace challenging but engaging while staying within the time format intended for the activity.

By the end of the development process, the activities we designed based on these goals largely adhered to the following principles:

- Awareness of students' pre-existing knowledge. Any experiences students have had relating to a given activity provide opportunities to conceptually build on those experiences. For example, when we run our activity about color mixing, students usually mostly know that television and mobile phone pixels come in red, green and blue, which simplifies introducing the idea of additive color mixing. However, students will often not know that blue and cyan are different colors and conflate the two, and activity leaders often need to address the difference between blue and cyan during the activity. Other ways we take students' knowledge levels into account are by (1) Including a few optional advanced challenges in the activity for students who complete the main part of the activity more quickly, and (2) answering questions by starting with a simple explanation and adding on details, perhaps on an individual basis, if more questions are asked.

- Activity segments building on previous segments. By introducing ideas one at a time and working from easy to hard concepts, we can reduce the cognitive demand on students while still giving them opportunities to apply what they learn as the activity progresses, and we can serve students at many different knowledge levels. ${ }^{3}$ For example, at one outreach event, we began by discussing the unique characteristics of lasers compared to common flashlights, demonstrated total internal reflection in water followed by transmission in fiber-optic cables, and finished by having students build an optical communication link through a fiber-optic cable.

- Open-ended problems and experimentation as part of an activity. Asking open-ended questions in the beginning of an activity, or giving students room to experiment to solve an interesting open-ended problem, can create a flexible environment within the activity that is conducive to discussion and learning. ${ }^{4}$ In all of the activities presented here, much or most of the time is spent in an environment of experimentation. 


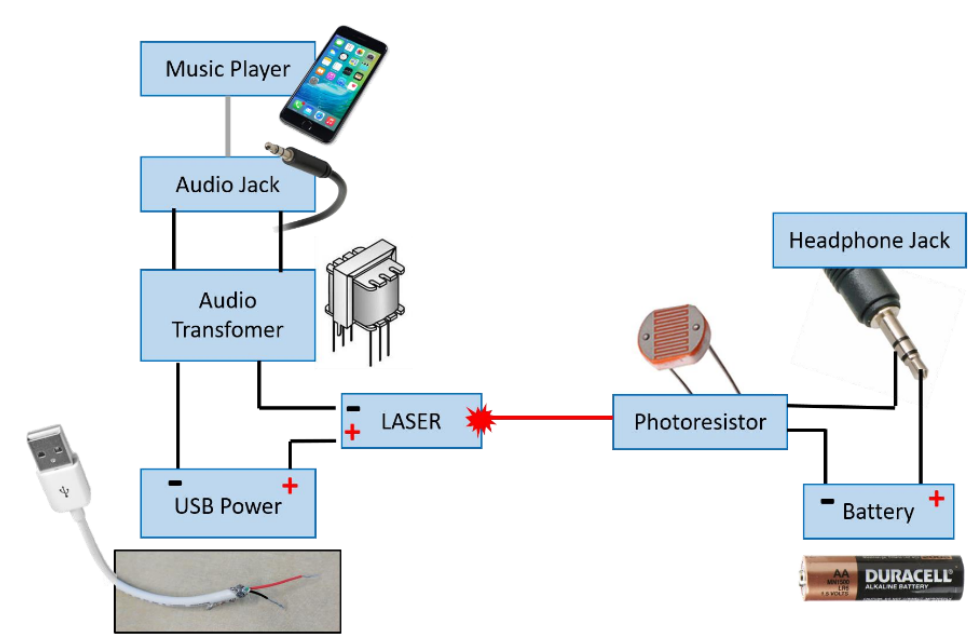

Figure 1. A schematic of the free-space optical link activity. We provide this schematic to students assembling the link. ${ }^{8}$

- Highlighting connections of subject matter to students' experiences and interests. By connecting the material to students' lives and interests, we aim to improve student learning ${ }^{5}$ and to spark a motivation to learn more that students can take with them when they leave. For example, an activity about photonic communications might relate to the internet and how we communicate with people who are far away, or an activity about solar cells might relate to energy efficiency, weather patterns, and climate change.

In practice, our activities generally start with an interesting question or "hook" based in experiences or concepts familiar to the students, and we allow time for ensuing group discussion. Following this, we may include one or two demonstrations illuminating concepts that might be new to students, building on their knowledge to facilitate the rest of the activity. We devote most of the time in the activity to a hands-on, self-directed challenge, e.g., building a small optical transceiver circuit. As students grapple with their challenge, activity leaders, usually but not necessarily graduate students, walk around the room and answer questions and help with troubleshooting on an individual basis.

In the last few minutes of the activity, we get the attention of the room so that we can summarize with all of the students what they learned and what they saw during the activity. For example, we may review of concepts from optics or photonics central to the activity, draw a link between the activity and a familiar real-world application, or say a few words about how the activity relates to the research interests of the activity leaders. We hope this reinforces the students' learning of the concepts they encountered during the activity, helps make the activity feel relevant to the real world, and stimulates students' interest in study and careers in the relevant fields.

\section{ACTIVITIES}

In the following sections, we describe some of our activities in more depth.

\subsection{Free-Space Optical Link}

This activity was adapted for high school students taking a class on the science of lasers and light that was taught over a series of five two-hour Saturday lessons at the UC Santa Barbara campus. ${ }^{6,7}$ Unlike the previous two activities, this activity as described typically runs for about one hour. In this activity, students build a transmitter circuit that transmits an audio signal, usually music from a student's or instructor's mobile phone, over light emitted by a laser or an LED to a receiver circuit that students also build that is connected to a pair of headphones. A schematic of this setup is provided in Fig. 1. 


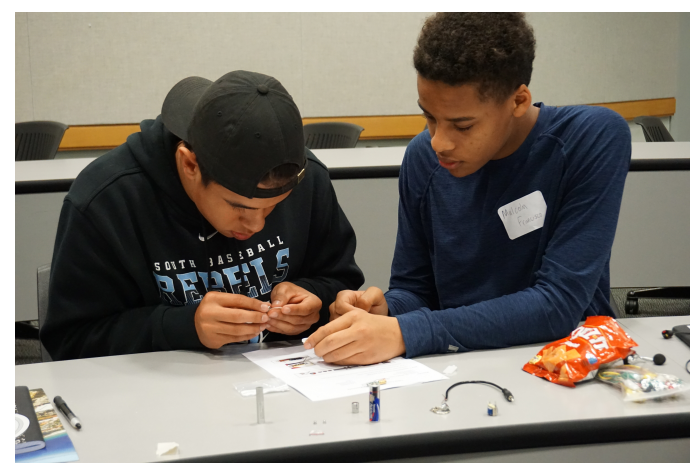

Figure 2. Students in the School for Scientific Thought (SST) Photonics Course working on the free-space optical communication link activity.

To begin the first lesson of the aforementioned class, we introduce the field of photonics and establish the concept of an optical communications link. Then, we provide materials and basic instructions and let students start to put together their optical links. From this point for the duration of the activity, instructors walk around the room to answer questions. We found most of the time required for this activity was taken up in this stage, with students (1) following the directions to build their optical links and (2) troubleshooting their built optical links for errors or a failed part. A photograph of students working on the activity is shown in Fig. 2

Once students build a working optical link, we invite students to place a fiber-optic cable between their laser and photoresistor, beginning a discussion on the benefits of waveguiding the laser light to communications. We also invite students to compare use of a laser with a blue LED as their light emitter. For each light emitter, students compare the quality of transmitted audio to, e.g., the distance between light emitter and photoresistor, or the ability to couple into a fiber-optic cable.

\subsection{Color-Mixing activity}

We developed an activity around color perception and the difference between additive and subtractive color mixing for science education events attended by middle school students and their families. ${ }^{9}$ The activity is 25-min in length, and self-contained-that is, there is no additional session during which knowledge or intuition is gained before or after the module. Before the activity, the desks of the classroom are arranged such that 2-3 families can cluster around the table. In addition, we put cyan, magenta, and yellow (CMY) dyes mixed in water, paintbrushes, and laminated pages with useful diagrams on the table. We also place posters with more useful diagrams around the classroom. The content of these posters is pictured in Figs. 3a and 3b.

We begin the activity with a hook: what colors are in the rainbow? This is a question that most young middle-school children (ages 11-14) are able to answer. As we ask this question, we demonstrate a rainbow by shining a white flashlight into a clear glass container filled with water and a compact mirror such that a rainbow forms on the ceiling. We follow up with the questions, what colors are not in the rainbow and how the colors in the rainbow being shown are formed. The main conclusion that students should draw is that the colors in the rainbow are included in white light. Some students are advanced enough to have heard this.

After this introduction, we give a five-minute mini-lecture on red, green, and blue (RGB) receptors in the eye and subtractive color mixing. We explain that by exciting the RGB receptors in different proportions, we can cause the brain to see different colors. For example, as we direct attention to Fig. 3a, we might say to students that while a yellow flower appears yellow because it reflects yellow light, when we see a yellow flower on a screen, it appears yellow because we are turning on tiny red and green lights, or pixels, in different strengths so that the brain interprets the combination as yellow.

This leads into the next segment of the activity which is on color printing (subtractive color-mixing). To motivate this, we draw attention to printer cartridges that we have placed around the room before the activity and ask students why color printers might use CMY and not RGB. We explain that the colors of the CMY color system block RGB colors individually. That is, cyan on a page absorbs red, but reflects blue and green; magenta absorbs green but reflects red and blue; yellow absorbs blue but reflects red and green. Therefore using the CMY 

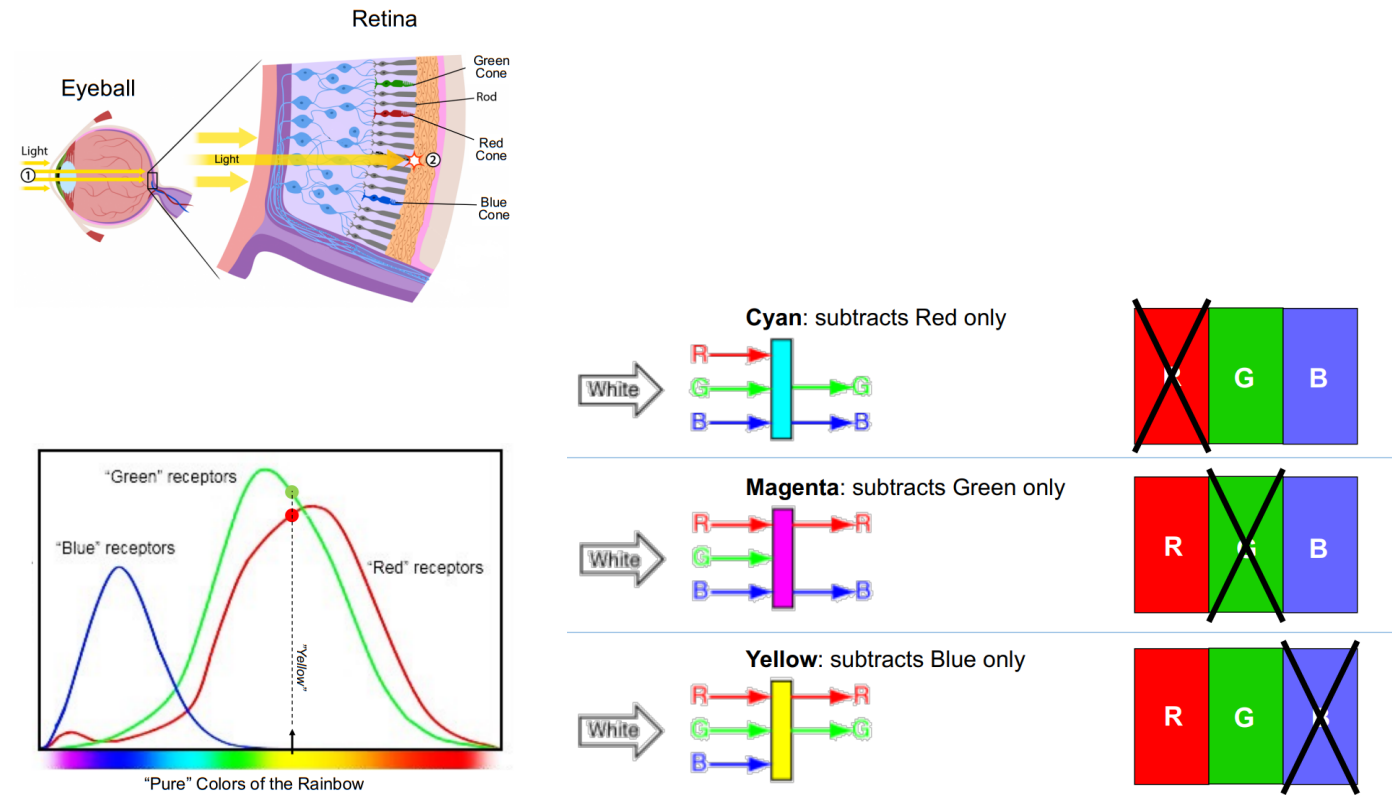

(a)

(b)

\section{Color Mixing!}

\section{Use your cyan, magenta, yellow palette to mix red, green, and blue:}

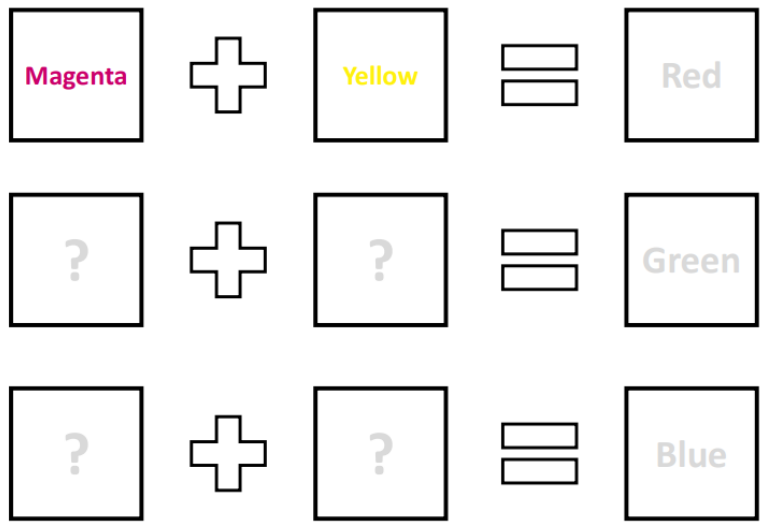

(c)

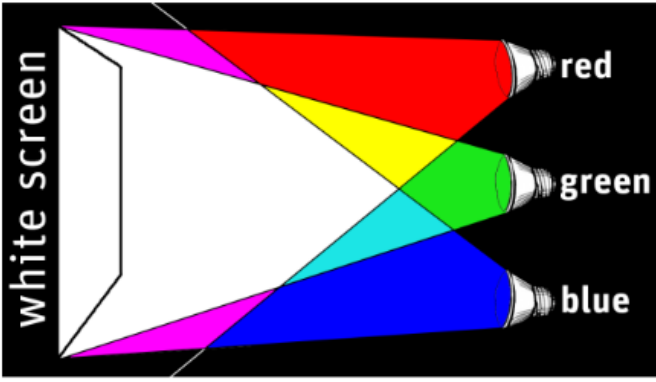

(d)

Figure 3. Handouts provided to students and their families attending the color mixing activity for the purposes of (a) explaining the function of rod and cone cells to human color perception, (b) showing how the colors cyan, magenta, and yellow used in subtractive color mixing relate to red, green, and blue, and (c) challenging students to solve subtractive color mixing problems. Additionally, (d) a diagram of the colored flashlights demonstration at the end of the activity. ${ }^{10}$ 

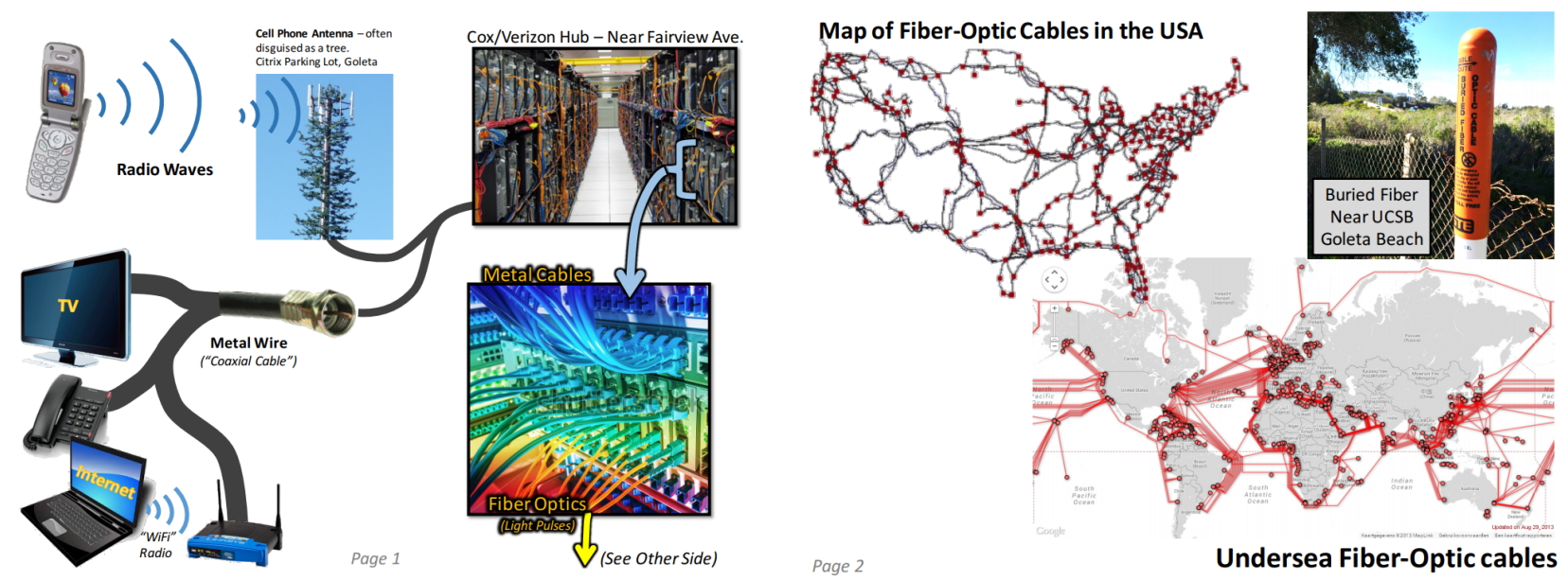

Figure 4. Handouts provided to students and their families attending the gelatin waveguides activity for the purpose of explaining the importance of lasers and fiber-optic cables to internet communications. ${ }^{11}$

color system one can control the amount and color of light that reaches the eye from a page by starting from white and individually reducing red, green, and blue, the opposite process compared to an electronic screen.

At this point, we pass out worksheets and challenge students to make red, green, and blue from the CMY dyes. The worksheet we pass out to students is pictured in Fig. 3c. If students and their families complete this activity quickly, we have an additional worksheet on which they can try to make so-called "secondary" colors such as orange, purple, and brown. During this portion of the activity, graduate student activity leaders circulate around the room to assist families who are completing the activity. One common mistake we see is that students often put cyan in the blue "answer", and think that the task is complete. It is important to point out the difference between cyan and blue. The blue in the RGB color system is much darker than what many people expect. We have also had instances of students knowing or discovering that they are colorblind. In this case, if the student is open to it, we use what the student has learned to discuss the origin of red-green colorblindness.

We wrap up the activity with a short activity on colored shadows. When it appears that students and their families are nearing completion of the activity, a graduate student volunteer will set up a white sheet (if a plain white wall is unavailable) and red, green, and blue flashlights next to each other with some lateral distance between them. This setup is pictured in Fig. 3d. We dim or turn off the lights in the classroom and turn on the colored flashlights. We ask that a volunteer step in front of the flashlights so that the students discover they see different colored shadows. We use this time to both review the difference between additive and subtractive color mixing while reinforcing the mechanism of subtractive color mixing.

\subsection{Gelatin Waveguides}

This is another self-contained 25-minute activity that we developed for science education events attended by middle school students and their families. ${ }^{9}$ The setup for this activity is similar: pages with useful diagrams are distributed, and other materials (fiber optic cables, laser pointers, pieces of gelatin) are distributed as needed during the activity. We find it best to hand out materials as they become relevant, so the students do not get distracted during preliminary segments. Before the activity, we set up a demonstration of total internal reflection by mixing water and a few drops of milk or half and half into a clear rectangular container, then testing with a green laser pointer for reflections and visibility of the laser light path through scattering. We also set up a fiber optic cable demonstration by making a few bundles of several fiber optic cables per bundle.

This activity begins with a hook: "What exactly happens when you send a text message or an e-mail?" Usually students volunteer some basic answers related to cellular phone towers, satellites, and sometimes data centers. After allowing some time for discussion, we use the images and illustrations pictured in Fig. 4 that were distributed to families at the beginning of the activity to lead up to explaining that communication on the 


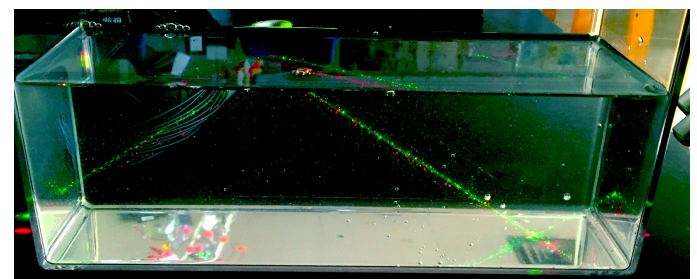

(a)

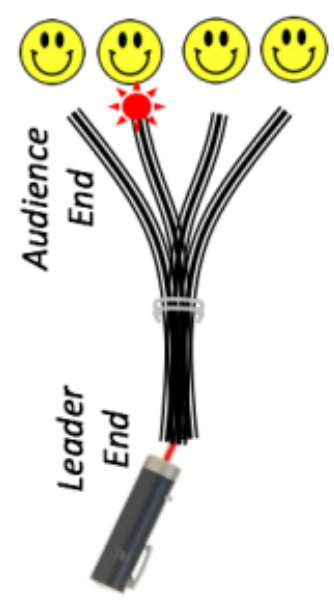

(b)

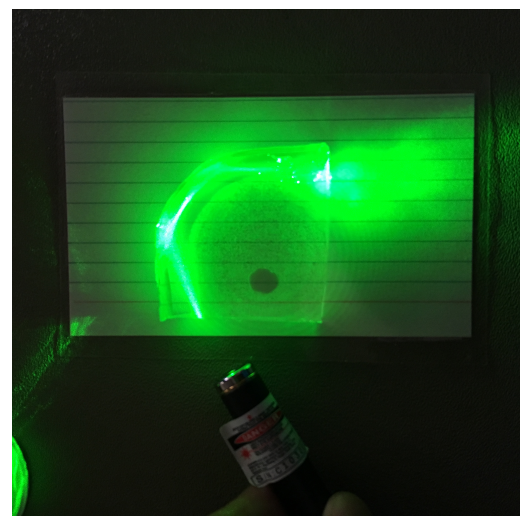

(c)

Figure 5. Demonstrations in the gelatin waveguides activity: (a) the total internal reflection demonstration used at the beginning of the activity; (b) a schematic of the fiber optic cable demonstration; (c) a partial solution to the challenge we put to participants to bend laser light 180 degrees.

internet largely relies on lasers, which transmit information in the form of light waves, and a global network of fiber-optic cables, which carry the information.

After this, we introduce a laser pointer to the classroom and ask the room: "What is special about a laser?" We find it helps to contrast the laser with a conventional flashlight like those that can be found on most mobile phone cameras today. Here, the main takeaways for students are that, unlike flashlights, lasers are very bright, travel in a straight line, and are one color. This is followed by a demonstration of total internal reflection. We motivate this demonstration by asking how to control light over long distances, e.g., to communicate with somebody on another continent. With the room lights off, we point our green laser into the milky water that was set up before the activity and allow students to observe that for sufficiently glancing reflections, all of the light is reflected within the water. This demonstration is pictured in Fig. 5a. We explain that some light is transmitted and some is reflected when it passes between two materials, e.g. air and water, and that if we find an angle where all of the light is reflected within a material, we can make a waveguide, or "light pipe," out of that material and use it to send the light a long distance.

Having explained total internal reflection, we begin a fiber optic cable demonstration by distributing the fiber optic cables at one end of our pre-assembled fiber optic cable bundles, with each family getting one fiber optic cable end, and each activity leader holding all of the fiber optic cables at the other end of the bundle. We explain that activity leaders will send a message through one of the fiber optic cables, without telling families which cable will be used. Activity leaders then send simple Morse code-like signals through the fiber optic cables, and families can observe the signal reaching their cables despite bends and turns in the fiber optic cable bundle. A simple schematic of this demonstration is included in Fig. 5b. At the end of this demonstration, we explain that, as in the tank of milky water, light can be trapped, or guided, in plastic fibers.

In the final part of the activity, we distribute pieces of gelatin (typically $2 " \times 2 " \times 1$ ") to each student. We challenge the students to form a shape out of their gelatin that can turn a laser beam 180 degrees, and then we pass out laminated flashcards to each student to use as a safe cutting edge. An example partial solution is pictured in Fig. 5c. As in the previous part of the activity, activity leaders walk around the room, answer questions, and guide students as they form their gelatin waveguides.

\section{RESULTS}

At the time of writing, we have held the color mixing activity at about twelve different outreach events, impacting an estimated 830 middle school students total. We have held the gelatin waveguides activity at about six different 
outreach events, impacting an estimated 380 students total. We have held the optical link activity at about six different outreach events, impacting about an estimated 140 students total.

Over the course of these events, we found that students were most engaged by the "challenge" portions of each activity which were open-ended and conducive to experimentation. To tackle or better understand their challenges, students would often make informed hypotheses, systematically explore all of their available options, and/or use another student's working solution as a starting point, and leaders would often draw similarities between students' experiences and the scientific research process. Having visual aids available helped activity leaders during explanation of key concepts, e.g. by using posters with illustrative figures and diagrams placed in prominent parts of the room for group discussion, or by providing this material on handouts to each student for one-on-one discussions. We also found that engaging in discussions in a Socratic manner wherever possible instead of explaining or lecturing was also conducive to eliciting student engagement.

We have also identified some common points of difficulty in the running of our activities. In particular for the short 25-minute activities, timing requires close attention, as it is easy for any given discussion or demonstration to run long and leave insufficient time for the more engaging open-ended challenge section. It is also easy to let the challenge section run to the end of the available time, instead of concluding by summarizing the activity to help students retain, and relate back to their own experiences and interests, what they learned about how the activity works.

\section{CONCLUSION}

In this paper we have described the principles by which we develop educational outreach activities: We aimed to design activities that were open-ended, as well as discussion and experiment based. We described activities based on gelatin waveguides, additive and subtractive color mixing, and optical communication links. We also communicated our findings from many events at which we held each of our activities: Most importantly, we found that activity sections in which students tackled open-ended challenges were most engaging for students.

We have made the directions and materials list for these activities available on our website for the general public. $^{12}$

\section{ACKNOWLEDGMENTS}

The authors would like to acknowledge the Center for Science and Engineering Partnerships at UC Santa Barbara and the IEEE Photonics Society for their support.

\section{REFERENCES}

[1] Hirokawa, T. and John, D., "Engaging and educating your local community with a photonics-related activity," IEEE Photonics Society Newsletter 32, 7-9 (2018).

[2] "NGSS for California Public Schools, K-12 - Science (CA Dept of Education)." California Department of Education, 25 April 2019 https://www.cde.ca.gov/pd/ca/sc/ngssstandards.asp. (Accessed: 1 May 2019).

[3] Pollock, E., Chandler, P., and Sweller, J., "Assimilating complex information," Learning and instruction 12, 61-86 (2002).

[4] Allen, D. E., Duch, B. J., and Groh, S. E., "Situated cognition and the culture of learning," New directions for teaching and learning 1996, 43-52 (1996).

[5] Brown, J. S., Collins, A., and Duguid, P., "Situated cognition and the culture of learning," Educational researcher 18, 32-42 (1989).

[6] Field, S. Q., "Chapter 7: Light and Optics - Build a laser communicator. Send your voice over a laser beam.." Science Toys, https://sci-toys.com/scitoys/scitoys/light/light.html. (Accessed: 1 May 2019).

[7] "School for Scientific Thought | CSEP | UC Santa Barbara." Center for Science and Engineering Partnerships | California NanoSystems Institute | UC Santa Barbara, 2019 http://sst-csep.cnsi.ucsb.edu/. (Accessed: 1 May 2019). 
[8] Stanton, E., Rosborough, V., and Hirokawa, T., "Lasercomm Demo New.pdf." UC Santa Barbara Connect Box, 9 March 2018 https://ucsb.box.com/s/8ev607z7147n81h3q7z3p5nm68u3fm1n. (Accessed: 1 May 2019).

[9] "Family Ultimate Science Exploration | Center for Science and Engineering Partnerships | California NanoSystems Institute | UC Santa Barbara." Center for Science and Engineering Partnerships | California NanoSystems Institute | UC Santa Barbara, 2019 http://csep.cnsi.ucsb.edu/programs/fuse. (Accessed: 1 May 2019).

[10] Rosborough, V., Hirokawa, T., Chan, P., Jin, W., and John, D. D., "Microsoft Word - FuSE Colors v1.docx." Center for Science and Engineering Partnerships | California NanoSystems Institute | UC Santa Barbara, 2017 http://csep.cnsi.ucsb.edu/sites/csep.cnsi.ucsb.edu/files/programs/fuse_ colors_v1_0.pdf. (Accessed: 1 May 2019).

[11] John, D. D., Ibsen, W., and Garza, M., "FUSE Photonics - Slide for Table v3.pptx." Center for Science and Engineering Partnerships | California NanoSystems Institute | UC Santa Barbara, 8 February 2016 http://csep.cnsi.ucsb.edu/sites/csep.cnsi.ucsb.edu/files/programs/fuse_photonics_ -_slide_for_table_v3.pdf. (Accessed: 1 May 2019).

[12] "Education." Photonics Society at UCSB, 2018 http://ips.ece.ucsb.edu/education.html. (Accessed: 1 May 2019). 